\title{
FATORES ASSOCIADOS AO EXCESSO DE PESO ENTRE MULHERES
}

\author{
Factores asociados al exceso de peso entre mujeres
}

Factors associated to overweight among women

\author{
Aline Cristine Souza Lopes ${ }^{1}$ \\ Mariana Carvalho de Menezes ${ }^{4}$
}

Alessandra Nunes Lima Reyes²

Cibele Comini César ${ }^{3}$

Luana Caroline dos Santos ${ }^{5}$

\section{RESUMO}

Objetivou-se caracterizar as usuárias e identificar os fatores associados ao excesso de peso em serviço público de Promoção à Saúde (SPS). Estudo transversal com todas as mulheres de SPS, denominado Academia da Cidade. Utilizaram-se variáveis antropométricas, consumo e hábitos alimentares, e relato de morbidades. Realizou-se análise descritiva e regressão linear múltipla $(p<0,05)$. Verificou-se entre as 262 entrevistadas elevada prevalência de excesso de peso $(78,7 \%)$ e hábitos alimentares inadequados. Foram associados ao aumento do índice de massa corporal: mastigação inadequada de alimentos ( $b=2,780$; $p<0,001)$, consumo insuficiente de vitamina $C(b=-2,700 ; p=0,039)$, ter risco para doenças cardiovasculares $(b=2,840$; $p<0,001)$ e dislipidemias $(b=2,292 ; p=0,003)$, e realizar dieta nos últimos seis meses $(b=2,216 ; p=0,006)$. A alta taxa de excesso de peso e seus fatores associados apontam para a necessidade de realizar estratégias de promoção e recuperação da saúde, focadas no cuidado integral dos indivíduos e comunidades, ainda que em SPS.

Palavras-chave: Consumo de alimentos. Doença crônica. Hábitos alimentares. Obesidade. Fatores de risco.

\begin{abstract}
The objective is to characterize the users of a public health promotion service (HPS) and identify factors associated with excess weight among these users. It's cross-sectional study among all women in a HPS. It was used anthropometric variables, food consumption and dietary habits, and reports of morbidity. Descriptive analysis and multiple linear regressions were performed $(p<0,05)$. A high prevalence of excess weight $(78,7 \%)$ and inadequate dietary characteristics were found among the 262 interviewees. The increase of body mass index correlated with inadequate food mastication ( $b=2,780$; $p<0,001)$; insufficient vitamin C intake ( $b=-2,700 ; p=0,039)$; risk of cardiovascular diseases ( $b=2,840 ; p<0,001)$ and dyslipidemia ( $b=2,292 ; p=0,003)$; and doing diets over the last six months $(b=2,216 ; p=0,006)$. Conclusion: The high rates of excess weight and associated factors show that there is a need to implement strategies for health promotion and recovery, focusing on comprehensive care for individuals and communities, even if at HPS.
\end{abstract}

Keywords: Food consumption. Chronic disease. Food Habits. Obesity. Risk Factors.

\section{Resumen}

Se objetivó caracterizar las usuarias e identificar los factores asociados al exceso de peso en el Servicio de Promoción a la Salud (SPS) Pública. Estudio transversal con todas las mujeres de SPS, denominado Academia da Cidade. Se utilizaron variables antropométricas, consumo y hábitos alimentarios, e informe de morbilidades. Se realizó regresión lineal múltiple $(p<0,05)$. Se comprobó entre las 262 entrevistadas elevada prevalencia de exceso de peso $(78,7 \%)$ e erróneos hábitos alimentarios. Fueron asociados al aumento del índice de masa corporal (IMC): masticación inadecuada de alimentos $(b=2,780 ; p<0,001)$, consumo insuficiente de vitamina $C(b=-2,700 ; p=0,039)$, tener riesgo para enfermedades cardiovasculares $(b=2,840$; $p<0,001)$ y dislipidemías $(=2,292 ; p=0,003)$, y seguir dieta en los últimos seis meses ( $b=2,216 ; p=0,006)$. Las altas tasas de exceso de peso y sus factores asociados indican la necesidad de crear estrategias de promoción y recuperación de la salud, dirigidas al cuidado integral de los individuos y comunidades, aunque sea en SPS.

Palabras Clave: Consumo de Alimentos. Enfermedad Crónica. Hábitos Alimenticios. Obesidad. Factores de Riesgo.

\footnotetext{
${ }^{1}$ Doutora em Saúde Pública, Professora Adjunta do Departamento de Enfermagem Materno-Infantil e Saúde Pública e do Programa de Pós-graduação em Enfermagem da Escola de Enfermagem da Universidade Federal de Minas Gerais, Grupo de Pesquisa em Intervenções Nutricionais (GIN). Belo Horizonte - MG. Brasil. E-mail: aline@enf.ufmg.br; ${ }^{2}$ Mestre em Saúde e Enfermagem pelo Programa de Pós-graduação da Escola de Enfermagem (EE) da Universidade Federal de Minas Gerais. Belo Horizonte - MG. Brasil. E-mail: alenlima@gmail.com; ${ }^{3}$ Doutora em Demografia, Professora Associada do Departamento de Estatística do Instituto de Ciências Exatas da UFMG, Observatório de Saúde Urbana e Grupo de Pesquisas em Epidemiologia da Faculdade de Medicina da UFMG. Belo Horizonte - MG. Brasil. E-mail: cibele_comini@yahoo.com.br; ${ }^{4}$ Nutricionista, especialista em Saúde Coletiva com ênfase em Epidemiologia (UFMG), Mestranda em Saúde e Enfermagem da UFMG. Grupo de Pesquisa em Intervenções Nutricionais. Belo Horizonte - MG. Brasil. E-mail: marysnut@gmail.com; ${ }^{5}$ Doutora em Saúde Pública, Professora Adjunta do Departamento de Enfermagem Materno-Infantil e Saúde Pública e do Programa de Pós-graduação em Enfermagem da Escola de Enfermagem da Universidade Federal de Minas Gerais, Grupo de Pesquisa em Intervenções Nutricionais (GIN). Belo Horizonte - MG. Brasil. E-mail: luanacs@ig.com.br.
} 


\section{INTRODUÇÃO}

Nas últimas décadas tem sido verificado aumento importante da obesidade, incluída no grupo das doenças e agravos não transmissíveis (DANT), com diferenças entre os sexos, assim como das dislipidemias, hipertensão arterial (HA) e diabetes mellitus ${ }^{1}$.

A obesidade e as demais DANT estão entre as enfermidades que mais sobrecarregam o sistema de saúde, com demandas crescentes por ações, procedimentos e serviços. No Brasil, as DANT foram responsáveis em 2007 por $72,0 \%$ dos óbitos, sendo que a morbimortalidade atingiu principalmente a população mais pobre, acarretando elevado custo social e impacto nos gastos com a saúde².

A obesidade apresenta etiologia multifatorial destacando-se como fatores de risco passíveis de modificação a alimentação não saudável e a inatividade física. No tocante à alimentação, modificações importantes têm sido observadas na dieta dos brasileiros nos últimos anos, com destaque para o aumento da ingestão de alimentos ultraprocessados e redução daqueles minimamente processados como frutas, verduras e legumes ${ }^{3}$. Ademais, estudos destacam associação desta doença com o consumo inadequado de nutrientes, sobretudo cálcio, vitamina $C$ e zinco, em virtude da participação desses na regulação de processos metabólicos ${ }^{4}$.

Reconhece-se que a investigação detalhada desses fatores envolvidos na gênese da obesidade permite identificar indivíduos ou grupos mais vulneráveis, bem como subsidiar o planejamento de medidas e estratégias de prevenção e controle deste agravo, favorecendo políticas e abordagens com maior resolutividade?

Apesar do impacto da obesidade sobre a saúde dos indivíduos e de suas inter-relações com alimentos e nutrientes, verifica-se escassez de estudos que relacionem os hábitos alimentares e consumo de macro e micronutrientes às elevadas prevalências de excesso de peso, principalmente entre indivíduos socialmente vulneráveis. Assim os objetivos desse estudo foram caracterizar as usuárias e identificar os fatores associados ao aumento do índice de massa corporal em Serviço de Promoção à Saúde.

\section{METODOLOGIA}

Este estudo foi realizado no município de Belo Horizonte (BH), Minas Gerais, que tem população estimada de 2.412.937 habitantes, $330,95 \mathrm{~km}^{2}$ e é dividido em nove Distritos Sanitários. Estes Distritos dispõem de serviços de Atenção Primária, as Unidades Básicas de Saúde (UBS), que atuam como porta de entrada do usuário no Sistema Único de Saúde.
Adicionalmente, cada distrito apresenta pelo menos um Serviço de Promoção à Saúde, denominado "Academia da Cidade", que propicia aos usuários de áreas de elevada vulnerabilidade social, atividade física (3 vezes/semana com duração de 60 minutos) e orientação nutricional individual e coletiva. Esses serviços foram implantados em 2006 visando aperfeiçoar a abordagem para as DANT no município. A primeira Academia instalada, em projeto piloto, foi a do Distrito Sanitário Leste, alvo do presente artigo ${ }^{5}$.

Trata-se de um estudo transversal com todas as mulheres com vinte anos ou mais de idade, que ingressaram na referida Academia da Cidade no período de fevereiro a setembro de 2007. Optou-se por este período de tempo por constituir a fase final da implantação da Academia, época em que ocorreram adaptações dos protocolos.

A coleta de dados foi realizada no ingresso do usuário no serviço por acadêmicos dos cursos de Educação Física e Nutrição, devidamente treinados.

Utilizou-se anamnese pré-testada com dados sociodemográficos, hábitos alimentares (número de refeições diárias; comer assistindo TV; "beliscar" alimentos entre as refeições; mastigação dos alimentos; consumo de nutrientes e realizar dieta nos últimos seis meses), consumo alimentar obtido pelo Recordatório Alimentar de 24 horas (R24) e Questionário de Frequência Alimentar (QFA) e morbidade referida (dislipidemia - colesterol ou triglicérides elevados, diabetes mellitus, HAS e doenças cardiovasculares) ${ }^{6}$. Ademais, medidas antropométricas foram aferidas.

A ingestão dietética dos indivíduos foi avaliada a partir do R24 com aplicação única e de QFA, construído a partir dos alimentos habitualmente consumidos pela população em questão, visando minimizar as limitações apresentadas pelos métodos. 0 QFA, referente aos últimos seis meses, contou com uma lista de 16 itens alimentares, sendo utilizados neste estudo aqueles relacionados ao excesso de peso. Adicionalmente, foram obtidos, por meio de relato dos usuários, dados sobre número de refeições diárias, mastigação, consumo de alimentos em frente à $T V$, ingestão de líquidos às refeições, dentre outros ${ }^{6}$.

As medidas antropométricas contemplaram peso, estatura, circunferências da cintura (CC) e quadril (CQ), mensuradas de acordo com a Organização Mundial de Saúde $(\mathrm{OMS})^{7}$. A partir destas medidas obteve-se 0 índice de massa corporal (IMC=peso/altura $\left.{ }^{2}\right)$ e a razão cintura/quadril (RCQ), classificados de acordo com os pontos de corte propostos pela OMS ${ }^{8}$ e o IMC para idosos, segundo a adequação proposta pelo Nutrition Screening Initiative .

Para análise dos dados, o consumo alimentar, obtido pelo R24, foi analisado com auxílio do software Dietwin 
profissional versão 2.0 (2008). Foram avaliadas a adequação qualitativa do consumo de calorias, macronutrientes, vitaminas (vitamina $\mathrm{A}, \mathrm{E} \mathrm{eC}$ ) e minerais (cálcio, ferro e zinco), conforme preconizado pelo Institute of Medicine ${ }^{10}$. Para energia, foram utilizadas as fórmulas específicas para o estado nutricional da usuária, com as respectivas variabilidades. Para os macronutrientes, adotaram-se os intervalos recomendados de distribuição relativa (AMDR - Acceptable Macronutrients Distribution Range), sendo considerado como insuficiente ou excessivo os valores abaixo ou acima deste intervalo, respectivamente. Para os micronutrientes, utilizaram-se os valores de necessidade média estimada (Estimated Average Requirement - EAR), sendo considerado consumo adequado os valores superiores à EAR e inferiores ao nível máximo de ingestão tolerável (Tolerable Upper Intake Level - UL). A adequação da ingestão de fibras também foi avaliada, considerando-se os valores de ingestão adequada (Adequate Intake - Al) propostos pelo IOM. Por fim, verificou-se o consumo de colesterol a partir da proposição da $\mathrm{OMS}^{11}$.

As variáveis de exposição foram definidas com base na literatura, incluindo hábitos alimentares e nutrientes relacionados ao excesso de peso. Assim, foram incluídas na análise exploratória as variáveis sexo e idade como variáveis de ajustamento; morbidade referida (dislipidemia - colesterol ou triglicérides elevados, diabetes mellitus, HA e doenças cardiovasculares) e variáveis dietéticas (número de refeições diárias; comer assistindo TV; "beliscar" alimentos entre as refeições; mastigação dos alimentos; consumo de nutrientes, de acordo com o R24 e de alimentos, segundo o QFA; e realizar dieta nos últimos seis meses). 0 IMC, na forma contínua, foi adotado como variável resposta neste estudo.

Realizou-se análise descritiva dos dados com cálculo das frequências. A análise univariada foi efetuada a partir de modelos de regressão simples considerando o IMC como variável desfecho.

Para construção do modelo linear múltiplo foi utilizado o método por passos (stepwise) backward, utilizando-se as variáveis que apresentavam plausibilidade biológica ou que apresentaram valor $\mathrm{pd} \leq 0,25 \mathrm{na}$ análise univariada. As variáveis que apresentaram pe $\geq 0,05$ foram retiradas uma a uma do modelo e consideradas definitivamente excluídas, se o decréscimo na explicação do desfecho não era estatisticamente significativo.

A significância do modelo final foi avaliada pelo teste $F$ da análise de variância e a qualidade do ajuste pelo coeficiente de determinação ajustado $\left(R^{2}\right)$. Foi verificada a possível ocorrência de interações significativas entre as variáveis que permaneceram no modelo final. Ademais, todos os pressupostos referentes ao modelo foram cuidadosamente avaliados e nenhum deles foi violado. 0 programa SPSS (Statistical Package for the Social Sciences) versão 17.0 for Windows foi utilizado para as análises.

Quanto aos aspectos éticos, o estudo foi aprovado pelos Comitês de Ética da UFMG (ETIC No 103/07) e da Prefeitura Municipal de Belo Horizonte/MG (ETIC Nº 087/2007).

\section{RESULTADOS}

Ingressaram no Serviço de Promoção da Saúde no período do estudo 300 indivíduos, 262 (87,3\%) mulheres. Dessas $24,8 \%$ eram idosas (e $\geq 60$ anos) e 79,2\% apresentavam excesso de peso. Adicionalmente, $27,1 \%$ e $45,8 \%$, respectivamente, apresentavam risco elevado e muito elevado de complicações metabólicas, segundo CC, e $35,1 \%$ risco para doenças cardiovasculares, segundo RCQ (Tabela 1).

Além da obesidade, a ocorrência de DANT foi alta, especialmente HA e dislipidemias, presentes em 48,0\% e 35,3\% dos participantes, respectivamente (Tabela 1).

Em relação ao consumo de alimentos, verificou-se baixo consumo diário de frutas, verduras e legumes $(24,8 \%)$, elevada ingestão diária de alimentos ricos em gorduras e açúcar como "banha animal" (9,9\%), frituras $(8,8 \%)$ e refrigerantes comuns $(8,4 \%)$. Adicionalmente, as mulheres apresentaram reduzido fracionamento das refeições ( $46,4 \%$ realizavam $\leq 3$ refeições diárias), alta frequência de comer assistindo TV $(56,1 \%)$, mastigação inadequada $(44,3 \%)$ e tentativas de redução de peso nos últimos meses (32,1\%) (Tabela 2).

Quanto ao consumo de calorias e macronutrientes, $48,8 \%$ relataram ingestão insuficiente de calorias e $22,7 \%$, de carboidratos, enquanto a ingestão de lipídios foi excessiva em $40,8 \%$ e de fibras insuficiente em $64,8 \%$ das usuárias. Em relação aos micronutrientes, destacouse consumo insuficiente de cálcio $(96,5 \%)$, zinco $(49,6 \%)$, ferro $(48,5 \%)$, vitamina $A(59,2 \%)$ e vitamina C $(60,6 \%)$, contrastando com o consumo excessivo de vitamina $\mathrm{E}(78,8 \%)$ (Tabela 3$)$.

Alguns dos fatores associados ao excesso de peso na análise univariada são apresentados na Tabela 4. Com relação aos fatores associados ao aumento do IMC na análise linear multivariada, verificou-se influência da mastigação inadequada de alimentos $\left(b_{1}=2,780\right.$; $p<0,001)$, consumo insuficiente de vitamina $C\left(b_{2}=-\right.$ $2,700 ; p=0,039)$, risco para doenças cardiovasculares mensurado pela $R C Q\left(b_{3}=2,840 ; p<0,001\right)$, presença de dislipidemias $\left(b_{4}=2,292 ; p=0,003\right)$ e realização de dieta nos últimos seis meses $\left(b_{5}=2,216 ; p=0,006\right)$ (Tabela 5). 
Excesso de peso entre mulheres: fatores associados

Tabela 1 - Características gerais de usuárias de Serviço de Promoção da Saúde.

\begin{tabular}{|c|c|c|}
\hline Variáveis & $\mathrm{n}$ & $\%$ \\
\hline \multicolumn{3}{|l|}{ Idade (anos) } \\
\hline $20-29$ & 24 & 9,2 \\
\hline $30-39$ & 45 & 17,2 \\
\hline $40-49$ & 58 & 22,1 \\
\hline $50-59$ & 70 & 26,7 \\
\hline$\geq 60$ & 65 & 24,8 \\
\hline \multicolumn{3}{|l|}{ Estado nutricional Adultos } \\
\hline Baixo peso & 10 & 4,5 \\
\hline Eutrofia & 54 & 24,5 \\
\hline Sobrepeso & 55 & 25,0 \\
\hline Obesidade grau I & 59 & 26,8 \\
\hline Obesidade grau II & 24 & 10,9 \\
\hline Obesidade grau III & 18 & 8,2 \\
\hline \multicolumn{3}{|l|}{ Estado nutricional Idosos } \\
\hline Baixo peso & 6 & 7,5 \\
\hline Eutrofia & 20 & 25,0 \\
\hline Sobrepeso & 54 & 67,5 \\
\hline \multicolumn{3}{|l|}{ Circunferência da cintura } \\
\hline Risco elevado de complicações metabólicas & 71 & 27,1 \\
\hline Risco muito elevado de complicações metabólicas & 120 & 45,8 \\
\hline \multicolumn{3}{|l|}{ Razão cintura/quadril } \\
\hline Risco para doenças cardiovasculares & 92 & 35,1 \\
\hline Hipertensão arterial & 121 & 48,0 \\
\hline Dislipidemia & 89 & 35,3 \\
\hline Diabetes mellitus & 39 & 15,5 \\
\hline Doença cardiovascular & 21 & 8,3 \\
\hline
\end{tabular}

Tabela 2 - Hábitos alimentares de usuárias de Serviço de Promoção da Saúde.

\begin{tabular}{lll}
\hline \multicolumn{1}{c}{ Variáveis } & $\mathrm{n}$ & $\%$ \\
\hline "Beliscar” alimentos entre as refeições & 138 & 52,7 \\
Comer assistindo TV & 147 & 56,1 \\
Não mastigar bem os alimentos & 116 & 44,3 \\
Número de refeições/diárias ( $\leq 3)$ & 121 & 46,4 \\
Realizar dieta nos últimos 6 meses & 84 & 32,1 \\
Consumo de frutas, verduras e legumes & & \\
Diário & 65 & 24,8 \\
Não diário & 197 & 75,2 \\
Leite e derivados* & & \\
Diário & 158 & 60,3 \\
Semanal & 63 & 24,0 \\
"Banha animal”* & & \\
Diário & 26 & 9,9 \\
Semanal & 13 & 5,0 \\
Frituras* & & \\
Diário & 23 & 8,8 \\
Semanal & 95 & 36,3 \\
Refrigerante comum* & & \\
Diário & 22 & 8,4 \\
Semanal & 115 & 43,9 \\
\hline Demas InViduos & & \\
\hline
\end{tabular}

* Demais indivíduos incluídos com a frequência denominada outras. 
Tabela 3 - Adequação do consumo de calorias e nutrientes de usuárias de Serviço de Promoção da Saúde.

\begin{tabular}{|c|c|c|}
\hline Variáve is & $\mathrm{n}$ & $\%$ \\
\hline \multicolumn{3}{|l|}{ Calorias } \\
\hline Insufic iente & 127 & 48,8 \\
\hline Excessivo & 49 & 18,8 \\
\hline \multicolumn{3}{|l|}{ Carboidrato } \\
\hline Insuficiente & 59 & 22,7 \\
\hline Excessivo & 23 & 8,8 \\
\hline \multicolumn{3}{|l|}{ Proteína } \\
\hline Insufic iente & 48 & 18,5 \\
\hline Excessivo & 1 & 0,4 \\
\hline \multicolumn{3}{|l|}{ Lipíio } \\
\hline Insufic iente & 16 & 6,2 \\
\hline Excessivo & 106 & 40,8 \\
\hline \multicolumn{3}{|l|}{ Fibras } \\
\hline Insuficiente & 167 & 64,2 \\
\hline Adequado & 93 & 35,8 \\
\hline \multicolumn{3}{|l|}{ Colesterol } \\
\hline Adequado & 216 & 84,7 \\
\hline Excessivo & 39 & 15,3 \\
\hline \multicolumn{3}{|l|}{ Vitanina A } \\
\hline Insuficiente & 154 & 59,2 \\
\hline Excessivo & 69 & 26,5 \\
\hline Tóxico & 10 & 3,8 \\
\hline \multicolumn{3}{|l|}{ Vitanina $E$} \\
\hline Insufic iente & 21 & 8,1 \\
\hline Adequado & 34 & 13,1 \\
\hline Excessivo & 205 & 78,8 \\
\hline \multicolumn{3}{|l|}{ Viranina $\mathrm{C}$} \\
\hline Insuficiente & 157 & 60,6 \\
\hline Excessivo & 88 & 34,0 \\
\hline
\end{tabular}

Tabela 4 - Modelos de regressão linear simples para mulheres, sendo o Índice de Massa Corporal a variável dependente.

\begin{tabular}{lccc}
\hline \multicolumn{1}{c}{ Variáveis explicativas } & Coeficiente & Valor $\mathbf{p}$ & $\mathbf{R}^{2}$ \\
\hline Sexo & 1,516 & 0,151 & 0,007 \\
\hline Idade & $-0,024$ & 0,332 & 0,003 \\
Doença cardiovascular & 0,662 & 0,612 & 0,001 \\
\hline Dislipidemia & 1,669 & 0,024 & 0,134 \\
\hline Hipertensão arterial & 0,991 & 0,166 & 0,007 \\
\hline Diabetes Mellitus & 0,397 & 0,687 & 0,001 \\
Risco para doenças cardiovasculares, segundo razão & 2,592 & $<0,01$ & 0,204 \\
\hline "Beliscar" alimentos entre as refeições & 0,482 & 0,493 & 0,002 \\
Comer assistindo TV & 1,405 & 0,047 & 0,014 \\
\hline Ter realizado dieta nos últimos 6 meses & 1,798 & 0,017 & 0,139 \\
\hline Não mastigar bem os alimentos & 2,855 & $<0,01$ & 0,235 \\
\hline Consumo diário de frutas, verduras e legumes & 0,303 & 0,718 & 0,021 \\
Consumo adequado de calorias & 0,251 & 0,741 & 0,000 \\
\hline Consumo adequado de carboidratos & 1,436 & 0,061 & 0,110 \\
\hline Consumo adequado de colesterol & 0,396 & 0,672 & 0,003 \\
\hline Consumo adequado de lipídios & 0,864 & 0,224 & 0,072 \\
\hline Consumo adequado de ácido graxo poli-insaturado & 2,506 & 0,026 & 0,131 \\
\hline Consumo adequado de cálcio & $-0,343$ & 0,847 & 0,003 \\
\hline Consumo adequado de fibra & $-0,262$ & 0,724 & 0,003 \\
\hline Consumo adequado de ferro & 0,274 & 0,542 & 0,036 \\
\hline Probabilidade de adequação do consumo de vitamina C & $-0,641$ & 0,601 & 0,003 \\
\hline Probabilidade de adequação do consumo de vitamina E & $-1,201$ & 0,485 & 0,002 \\
\hline Probabilidade de adequação do consumo de vitamina A & 0,471 & 0,822 & 0,003 \\
\hline Probabilidade de adequação do consumo de zinco & $-0,311$ & 0,837 & 0,003 \\
\hline Nota: R2 - Coeficiente de determinação & & & \\
\hline
\end{tabular}


Tabela 5 - Modelo final de regressão linear múltipla para mulheres, sendo o Índice de Massa Corporal a variável dependente.

\begin{tabular}{lccc}
\hline \multicolumn{1}{c}{ Variáveis } & Coeficiente & IC 95\% & Valor p \\
\hline Intercepto & 27,924 & $(26,185 ; 29,663)$ & $<0,01$ \\
Não mastigar bem os alimentos & 2,780 & $(1,318 ; 4,241)$ & $<0,01$ \\
Probabilidade de adequação do consumo de vitamina C & $-2,700$ & $(-5,264 ;-0,135)$ & 0,039 \\
Ter risco para doenças cardiovasculares, segundo razão & 2,840 & $(1,329: 4,352)$ & $<0,01$ \\
cintura/quadril & & & \\
Ter dislipidemia & 2,292 & $(0,764 ; 3,821)$ & 0,003 \\
Ter realizado dieta nos últimos 6 meses & 2,216 & $(0,658 ; 3,775)$ & 0,006 \\
\hline
\end{tabular}

Nota: Coeficiente de determinação $\left(R_{\text {aujstado }}^{2}\right)=0,147$. IC: intervalo de Confiança. RCQ: Razão Cintura/Quadril.

\section{DISCUSSÃO}

Os resultados revelaram prevalências elevadas de excesso de peso e DANT entre os usuários do Serviço de Promoção da Saúde, sendo os fatores associados ao aumento do índice de massa corporal, hábitos alimentares não saudáveis, risco e presença de doenças, e a realização de dieta nos últimos seis meses. Esses fatores, em sua maioria, são passíveis de modificação por meio da adoção de uma alimentação mais saudável associada à prática de exercício físico.

A prevalência de excesso de peso identificada neste estudo foi bastante superior àquela verificada por estudos nacionais ${ }^{1}$, provavelmente devido às características do serviço em questão. Apesar da Academia da Cidade ter como principal objetivo a promoção da saúde, muitos usuários a buscam espontaneamente em virtude de condições insatisfatórias de saúde ou por encaminhamento realizado pelos profissionais das Unidades Básicas de Saúde.

Destaca-se, assim, que em um espaço no qual a promoção da saúde deveria ser prioridade, o tratamento de doenças ainda se faz uma urgência. Tal fato reporta para a necessidade da íntima integração entre a Academia da Cidade e as Unidades Básicas de Saúde da área de abrangência, visando o cuidado integral da saúde dos usuários de acordo com a vocação dos serviços.

Estudos nacionais têm demonstrado o comportamento epidêmico do sobrepeso e da obesidade, explicado como resultante de fatores individuais, ambientais e sociais ${ }^{8}$. Nesse estudo verificou-se, pela análise de regressão linear múltipla, que o aumento do IMC associou-se aos hábitos alimentares não saudáveis como não mastigar bem os alimentos e o consumo insuficiente de vitamina C; ao risco de desenvolver doenças cardiovasculares, avaliado pela RCQ; à presença de dislipidemia; e à realização de dieta nos últimos seis meses.

A mastigação inadequada pode favorecer $\mathrm{o}$ aumento do IMC em virtude de baixos estímulos à saciedade, tendo em vista que sua ativação se dá por estímulos desencadeados por impulsos nervosos nos proprioceptores musculares excitados durante a mastigação ${ }^{12}$. Um estudo conduzido por Teichmann et al..$^{13}$ visando identificar a prevalência e os fatores de risco para sobrepeso e obesidade entre as mulheres de São LeopoldoRS identificou maior risco de obesidade entre aquelas que relataram fazer as refeições de forma rápida, o que pode comprometer a qualidade da mastigação.

Já o consumo de vitamina $C$ pode se relacionar a alterações no IMC devido à participação dessa vitamina na biossíntese da carnitina. Na literatura, destaca-se relação entre a concentração de vitamina $\mathrm{C}$ e intracelular de carnitina e oxidação de gordura. Assim, o consumo insuficiente de vitamina Ce, consequentemente, a sua concentração plasmática reduzida associam-se à diminuição da oxidação da gordura, podendo afetar a composição corporal ${ }^{4}$.

A composição corporal não foi mensurada diretamente neste estudo, mas medidas de circunferências e a razão cintura/quadril sugeriram elevada adiposidade abdominal e riscos metabólicos envolvidos ${ }^{7}$. A associação entre a razão cintura/quadril e o aumento do IMC denota a possibilidade do seu uso como critério de risco para triagem e tratamento do excesso de peso.

A associação encontrada entre dislipidemia, realizar dieta nos últimos seis meses e 0 aumento do IMC pode ser explicada pelo tipo de estudo utilizado, seccional, o qual a causalidade não pode ser avaliada devido à ausência de temporalidade. Entretanto, a dislipidemia pode constituir um importante preditor de complicações futuras do excesso de peso entre os indivíduos, tendo em vista o seu envolvimento com a ocorrência de desordens cardiovasculares e outras DANT ${ }^{14}$.

Por sua vez, a restrição do consumo calórico também pode se relacionar a episódios de comer compulsivo ${ }^{15}$. Observase entre indivíduos que se impõem controle alimentar restritivo a ocorrência do processo de "desinibição", ou seja, o indivíduo tende a "liberar" a ingestão calórica em resposta a determinados estímulos, podendo ocasionar episódios de comer compulsivo, por ser difícil sustentar a restrição energética por tempo prolongado. Ademais, escolhas alimentares ou adaptações fisiológicas em resposta aos episódios de escassez alimentar podem aumentar a gordura corporal ${ }^{16}$. Corroborando os achados, em um estudo realizado com amostra representativa de 570 adultos de Salvador/BA, observou-se associação entre adotar dieta para perda ponderal e excesso de peso, entre mulheres ${ }^{17}$. 
Apesar de não associadas ao aumento do IMC, destacaram-se neste estudo inadequações alimentares importantes que podem favorecer a ocorrência de DANT, como o consumo excessivo de lipídios e insuficiente de fibras, corroborado pela baixa prevalência do consumo diário de frutas, legumes e verduras ${ }^{8}$.

Em relação ao consumo de micronutrientes, destacamse as altas prevalências de consumo insuficiente de cálcio, ferro, zinco, vitaminas A e C. Estes achados são concordantes com aqueles encontrados pelo estudo nacional Pesquisas de Orçamentos Familiares (2008-2009)'1. Tais resultados são desfavoráveis ao considerar a ocorrência de DANT, tendo em vista o efeito antioxidante das vitaminas A na prevenção do câncer e doenças cardiovasculares; a ingestão insuficiente de ferro e redução da imunidade, da produtividade e desempenho intelectual; além da inter-relação da ingestão de cálcio, zinco e vitamina $\mathrm{C}$ com controle hormonal e alterações metabólicas ${ }^{4}$.

Diferindo desses resultados, verificou-se consumo excessivo de vitamina E. Há evidências da associação entre a ingestão excessiva deste nutriente com efeitos protetores para doenças do coração ${ }^{14}$. No entanto, ressalta-se que o consumo excessivo de vitamina $\mathrm{E}$ observado neste estudo pode se relacionar ao consumo excessivo de óleo vegetal relatado pelos usuários, sendo considerado um fator dietético aterogênico ${ }^{3}$.

Apesar de destacar resultados importantes, o estudo apresenta algumas limitações que devem ser ponderadas. A primeira é intrínseca ao seu delineamento, por se tratar de um estudo transversal e não possibilitar a identificação da relação temporal entre causa e efeito. Adicionalmente, a realização de apenas um único R24 também pode ser um fator limitante tendo em vista que um único dia pode não refletir a ingestão real do indivíduo. № entanto, com o intuito de minimizar esse problema, foi realizado o QFA, visando captar a variabilidade qualitativa da dieta.

Esse estudo, ao identificar os fatores associados ao aumento do índice de massa corporal entre participantes de Serviço de Promoção da Saúde, com destaque para aqueles com influência da dieta, agrega informações importantes para a compreensão da dinâmica destes novos serviços e do processo de cuidar que envolve a obesidade e demais DANT. Ademais, os resultados reforçam a importância da modificação dos modos de vida com vistas à redução de agravos à saúde, corroborando a criação de novas estruturas no SUS para o melhor enfrentamento do novo perfil epidemiológico vivenciado.

\section{CONCLUSÃO}

As altas taxas de excesso de peso e os fatores associados encontrados apontam para a necessidade da realização de estratégias de promoção e recuperação da saúde, focadas no cuidado integral dos indivíduos e comunidades, mesmo em serviços de promoção da saúde.
Lopes ACS, Reyes ANL, César CC, Menezes MC, Santos LC

\section{AGRADECIMENTOS}

À Fundação de Amparo de Minas Gerais, pelo financiamento do projeto, sob o processo de número CDS APQ0376-4.08/07.

Colaborações: Lopes ACS contribuiu na concepção, análise e interpretação dos dados, elaboração do trabalho e revisão crítica do conteúdo e aprovou a versão final; Lima AN contribuiu na concepção, análise e interpretação dos dados, auxiliou na elaboração do trabalho e aprovou a versão final; César CC contribuiu na elaboração, análise e interpretação dos dados e revisão crítica do conteúdo e aprovou a versão final; Menezes MC contribui na redação do manuscrito e sua aprovação final; Santos LC contribuiu na interpretação dos dados, elaboração do trabalho e revisão crítica do conteúdo e aprovou a versão final.

\section{REFERÊNCIAS}

1. Instituto Brasileiro de Geografia e Estatística-IBGE. Pesquisa de orçamentos familiares 2008/2009. Rio de Janeiro; 2010.

2. Schmidt MI, Duncan BB, Azevedo e Silva G, Menezes AM, Monteiro CA, Barreto SM, et al. Chronic non-communicable diseases in Brazil: burden and current challenges. Lancet. 2011 Jun; 377 (9781): 1949-61.

3. Monteiro CA, Levy RB, Claro RM, Castro IRR, Cannon G. Increasing consumption of ultra-processed foods and likely impact on human health: evidence from Brazil. Public Health Nutr. 2011 Jan; 14(1): 5-13.

4. Johnston CS, Beezhold BL, Mostow BO, Swan PD. Plasma Vitamin C is inversely related to body mass index and waist circumference but not to plasma adiponectin in nonsmoking adults. J Nutr. 2007 Jul; 137(7): 1757-62.

5. Dias MAS, Lopes ACS, Bicalho K, Mourão M, Alves MN, Evangelista PAR, et al. In: Júnior Magalhães HM. Promoção à saúde e articulação intersetorial. Desafios e inovações na gestão do SUS em Belo Horizonte: a experiência de 2003 a 2008. Belo Horizonte: Mazza Ed; 2010. p. 63-100.

6. Lopes ACS, Santos LC, Ferreira AD. Atendimento nutricional na atenção primária à saúde: proposição de protocolos. Nutr Pauta. 2010 maio; 101(1): 40-44

7. World Health Organization-WHO. Physical status: use and interpretation of anthropometry. Geneva; 1995.

8. World Health Organization-WHO. Obesity: preventing and managing the global epidemic. Geneva; 1998.

9. Nutrition Screening Initiative- NSI. Nutrition interventions: manual for professionals caring for older americans. Washington, DC; 1992. 
10. Institute of Medicine(USA). Food and nutrition board, dietary reference intakes research synthesis: workshop summary. Washington, DC; 2006.

11. World Health Organization-WHO. Interim summary of conclusions and dietary recommendations on total fat $\&$ fatty acids. FAO/WHO. Expert Consultation on fats and fatty acids in human nutrition. Geneva; 2008.

12. Apolinário RMC, Moares RB, Motta AR. Mastigação e dietas alimentares para redução de peso. Rev CEFAC. 2008 mar; 10(2): 191-99.

13. Teichmann L, Olinto MTA, Costa JSD, Ziegler D. Fatores de risco associados ao sobrepeso e a obesidade em mulheres de São Leopoldo, RS. Rev Bras Epidemiol. 2009 set; 9(3): 360-73.

14. Castro LCV, Franceschini SCC, Priore SE, Pelúzio MCG. Nutrição e doenças cardiovasculares: os marcadores de risco em adultos. Rev Nutr. 2004 set; 17(3): 369-77.

15. Marín-Guerrero AC, Gutiérrez-Fisac JL, Guallar-Castillón P, Banegas JR, Rodrígues-Artalejo F. Eating behaviours and obesity in the adult population of Spain. Br J Nutr. 2008 Nov; 100(5): 1142-48.

16. Bernardi F, Cichelero C, Vitolo MR. Comportamento de restrição alimentar e obesidade. Rev Nutr. 2005 fev; 18(1): 85-93.

17. Oliveira LPM, Assis AMO, Silva MCM, Santana MLP, Santos NS, Pinheiro SMC, et al. Fatores associados a excesso de peso e concentração de gordura abdominal em adultos na cidade de Salvador, Bahia, Brasil. Cad Saude Publica. 2009; 25(3): 570-82. 